\title{
Supporting Scenario-Based Product Design and Its Adapters: An Informal Framework for Scenario Creation and Use
}

\author{
Irene Anggreeni and Mascha van der Voort \\ University of Twente, Laboratory of Design, Production \& Management, \\ P.O. Box 217, 7500 AE Enschede, The Netherlands \\ \{i.anggreeni,m.c.vandervoort\} @utwente.nl
}

\begin{abstract}
This paper proposes a support tool for designers who have realized the potential benefits of using a scenario-based approach, yet need a more concrete guidance for its implementation. As an informal framework for scenario creation and use, the tool supports three areas: (1) documenting the large amount of design information, (2) making sense of and summarizing the information by creating scenarios, and (3) sustaining an integrated use of the scenarios throughout the design process. By making explicit and supporting the process of using scenarios, we expect that designers can better structure their design information, communicate their ideas and confirm their rationales. Our ongoing work includes the verification of the tool concept and the development of a testable prototype.
\end{abstract}

Keywords: scenario based design, product design, scenario based product design, scenario generation, design tool.

\section{Introduction}

Designing consumer products nowadays is becoming more complex. In the effort to accommodate diverse users and their use situations, designers have to take into account a product's dynamic use situations [1] to ensure a good usability in all possible settings, for varied user characteristics, etc. To cope with different design aspects, design teams often have to integrate different disciplines and backgrounds, which in turn poses a challenge in communication. Stories have been an inseparable part of communication as it is natural for us to communicate by telling them. Scenarios in this research context refer to a more structured, exploited version of stories. Whether expressed or not, scenarios are inherent in design processes and play a special role as a communication tool. Their use has been made more explicit with the advance of scenario-based design (SBD) within software development [2-5] as well as within product design domain [6-8]. Nevertheless, SBD as a methodology remains largely unguided and is perceived as an eclectic combination of existing design techniques and appropriate scenario uses. Consequently, industrial adapters of SBD are often discouraged because they have to speculate their own eclectic approach in applying 
scenarios. A concrete and practical guidance in carrying out scenario creation and use will support them to conduct SBD effectively and efficiently.

There exist formal frameworks that integrate scenarios in requirements engineering (e.g. [9-11]) as well as tools that explicitly address the generation and management of scenarios (e.g. [12-15]). Nevertheless, these frameworks and tools tend to focus on a specific activity or phase within the development process, and not the complete design project. To actively support SBD adapters, there needs to be a framework that sustains an all-round scenario-based approach throughout the design process, with the in-between results being optimally used. This framework should also fit in with the storm of creativities and dynamics of product designers. Such support is currently not available to the industry. We propose an informal framework that guides sustainable information documentation and scenario generation. This paper will explain how a scenario generation support tool could help an easier transition to a scenario-based design practice.

\section{Approach: Adaptation of SBD Theories into Design Practice}

Existing scenario-based design frameworks are rather theoretical and not proven in practice. Dealing with different types of design projects, practitioners nowadays have to take an eclectic approach to apply scenarios where they see fit. As the first step in our research, we have studied literature to get acquainted with the developing state of scenario-based design. An overview of scenario uses in the design domain has been formulated into a scenario classification [16]. This classification has helped us to pinpoint the often-not-explicit process of using scenarios in our target group users. By making the knowledge transparent to both researchers and designers (our target users), we were able to elicit their underlying reasons and concerns in using scenarios in real projects. The designers' involvement has given insights into their practice-grounded requirements that enable the extraction of criteria for the support tool.

Our proposed tool targets product designers in small- to medium-sized design companies. Interaction among designers in this target group is direct and transparent, which makes it easier for us to observe and subsequently support the information exchange. Contacts with designers from different backgrounds (roles, experiences, project types) have been established. From a series of workshops and questionnaires involving our contacts, we found the following challenges in design practices: (1) documenting the vast amount of relevant design information, (2) organizing the information into manageable and meaningful pieces and (3) making sure important and relevant information is traceable and useful to support design decisions. Creating scenarios is a partial answer to these challenges. However, due to a lack of guidance the created scenarios are often scattered and their use not fully integrated in the process. Furthermore, designers also wish for a more efficient and stimulating way of documenting information and composing scenarios.

We therefore focus on the backbone activities of SBD, i.e. the documentation of design information as scenario building blocks and the creation of scenarios to keep this information alive. Designers wish 'guidance' instead of 'prescribed steps' in 
conducting a scenario-based approach. The flexibility of our proposed framework will give room for a living approach which is strongly connected to each design phase.

\section{Functionality}

A structure for documenting design information is an essential part of the tool. It could then guide designers to create meaningful scenarios that give a better picture of the various use situations. Furthermore, dealing with scenarios and requirements, the design team and stakeholders should be able to get an overview as well as detailed views of information during decision making.

To introduce the actors involved in our support tool, we summarize their roles and interests in a design project. We assume that these typical roles in a design project are generic enough and can be recognized in different project settings.

- Project manager (PM) is the one who manages the course of a design project. During the initial stage of the project, PM is responsible for defining an agreement with the client and possibly other stakeholders on subjects such as project timeframe and budget. An important part of this agreement is the design approach which the design team will use to accomplish the assignment. As it directly relates to cost, the client could wish to remove parts of the proposed approach to cut expenses.

- Client is the one giving an assignment to the design company. The client has the biggest interest in the design project in terms of business profit. During the initial stage of the project, the client defines a clear orientation and project scope with the design team. He also has rich information, for instance about the target users/buyers, a rough product idea and relevant technologies, potential sources of information (e.g. people, documents, standards or regulations) and competitor products of similar line. Therefore, even in the early stage predefined requirements are often already constructed. This initial information however could have been assumed by the client and therefore needs to be verified through further research.

- End-users are the persons or professionals who are going to use the product. They have the domain skill and knowledge that is resourceful towards the design. The design team needs to obtain this tacit information to be able to design a useful product. A variety of participatory design techniques/methods can be used to involve these people in design activities.

- Designers are members of a design team and could be of diverse functions such as engineers, software developers, usability specialists, business analysts, ergonomic experts, etc. They conduct design activities to fulfill the assignment as agreed by the PM and client. Even with the design approach already defined, designers are more stimulated when they have freedom to employ their multitude of skills, creativity and perspectives in executing design techniques. Therefore despite their different backgrounds and functions, we refer to these creative problem-solvers in a design project as simply designers. 
The set of scenarios below explains briefly the context for which the tool offers support. The functions described in this paper are select ones that highlight the scenario identification and generation, and therefore do not show the complete functionality. In each section, a set of illustrations and intended use scenarios are presented to portray the interaction with the tool. Figure 1 shows the first step using the tool, in which its components are introduced by means of an example dealing with the design of a bike accessory.

\begin{abstract}
A new project Urban Mobility kicks off
The project 'Urban Mobility' has reached its final agreement with client Speeda Inc. The design team, lead by Bob, is quickly preparing a set of action plans to tackle the initial phase. For instance, Marie is visiting an exhibition of bicycle latest gadgets/technology to survey the market. John, another designer in the team, is recruiting current users of bicycle accessories for carrying luggage, to get insights on their way of using existing products.

During the focus group session...

John invites two users to a focus group session. The session takes place in an informal setting at the users' place. The discussion deals with the users' daily activities, situations and events which occur around these activities and the users' wishes and needs for improvement.

At the exhibition...

Marie collects brochures, takes pictures and notes comments about the latest bicycle gadgets and technologies that may be relevant with the project. She also learns about the benefits and limitations of the latest products from talking with the people at the exhibition.
\end{abstract}

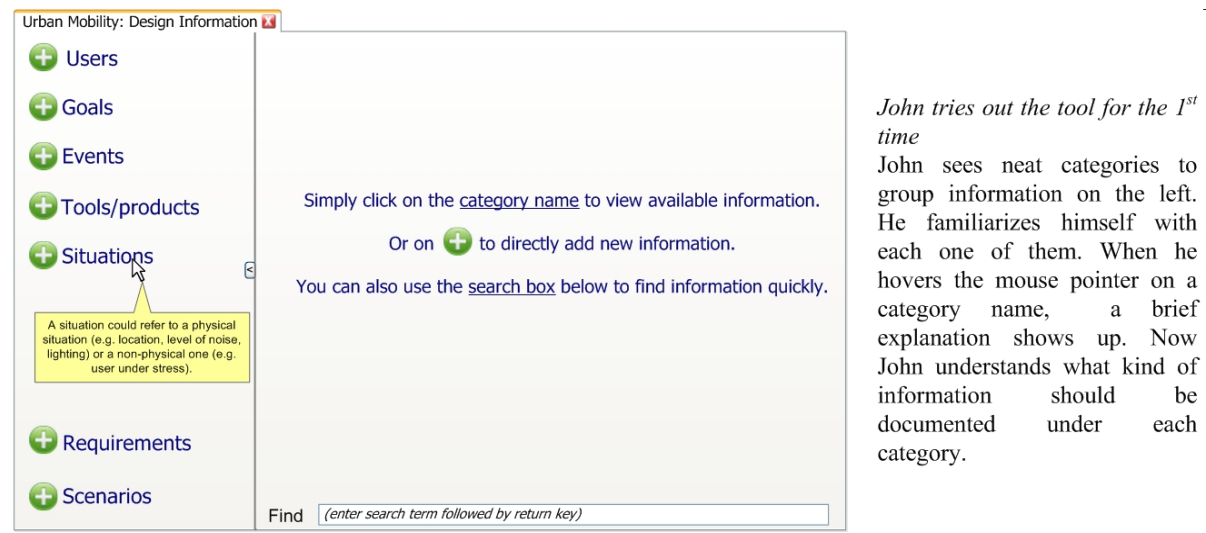

Fig. 1. The welcome screen provides simple categories that guide the design team to document relevant information

\title{
3.1 Design Information Documentation: Identifying Scenario Elements
}

During the initial phase of a design process, understanding the global view of information and making sure the validity of all information up to now are essential. A combination of common design methods/techniques such as ethnography, observation, focus group, interview, participatory design can be used to capture all elements 
of product use, e.g. users, their goals, current products they use, their tasks/activities and events that might happen during product use.

With the various sources of information, it can be overwhelming for design team members to grasp all relevant aspects of the use situations. The diverse media (e.g. video/audio recording, photos, brochures, mockup models) in combination with vast amount of information could result in a lack of organization, which makes information less traceable. Designers wish for a more flexible and stimulating way to sort and record information pieces while still keeping them coherent.

The tool provides a template to guide designers to classify their information in categories inspired by scenario elements. The existence of a template will inform design team members of the priority information. Fig. 2-4 show how the designers register information of different categories. By organizing the information early on, the tool could sustain designers' motivation and enthusiasm while documenting.

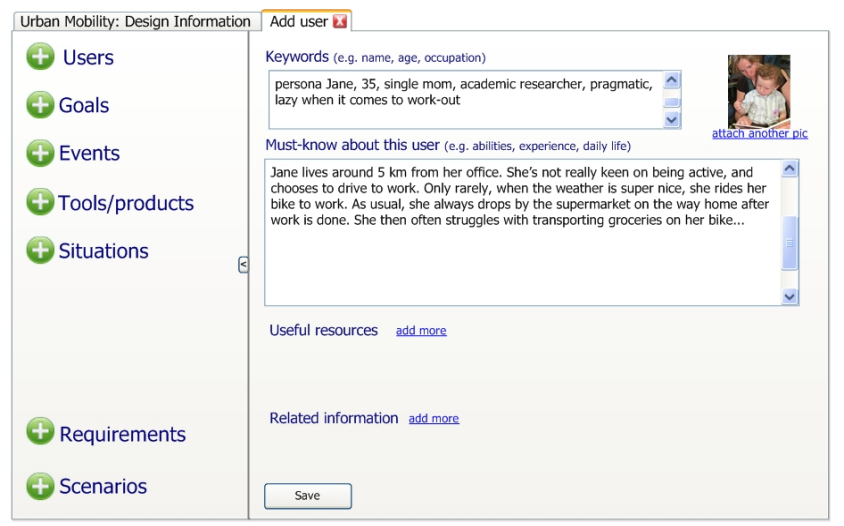

John creates a user profile using the scenario support tool John summarizes what he has learned from the focus group session. Both participating users are quite similar in the way they are using bike pannier. Therefore John creates a fictive user Jane that combines their characteristics.

Fig. 2. Adding a user element without much prescribed steps; the designer is free to include whatever information he or she feels necessary
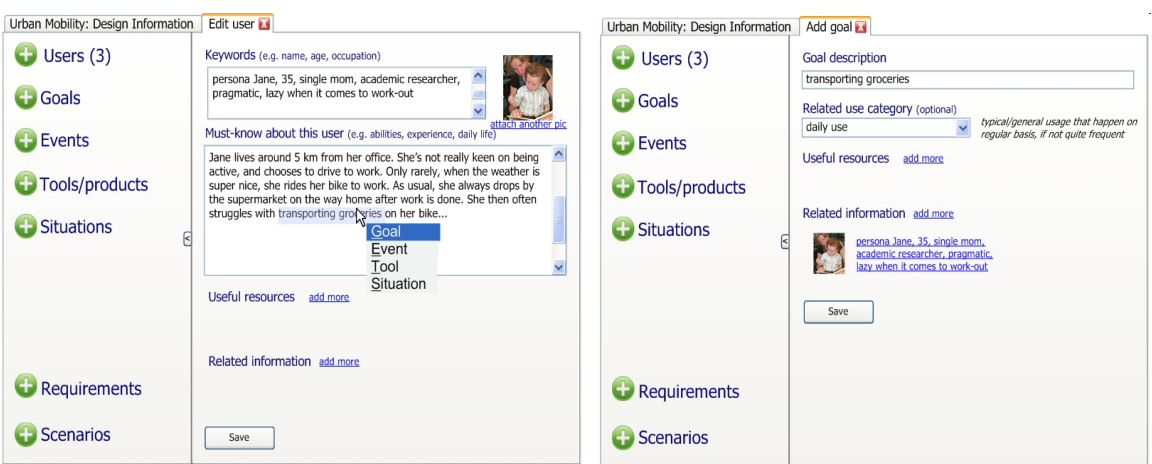

John identifies and extracts other scenario elements from a user profile

John revisits the user profile Jane and breaks it down further by scanning for other useful information. For instance, he notices a goal 'transporting groceries' is implicit in the user description. He marks the piece of text and by right-clicking, could register it as an explicit goal. The new goal keeps a reference to the user profile Jane as its source of origin.

Fig. 3. New scenario elements can be denoted from existing ones; their relations are explicitly maintained for better traceability 


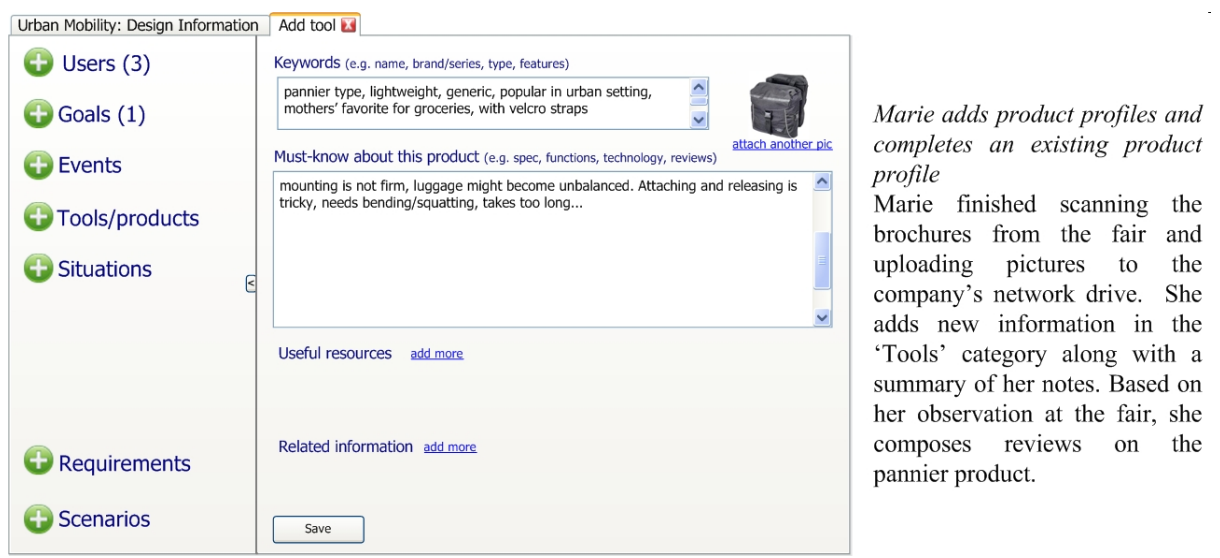

Fig. 4. An existing product can be added as a tool scenario element; the designer can also freely add a product concept in progress to make mention of its characteristics

\subsection{Design Information Processing: Scenario Building and Requirements Extraction}

A design team is responsible to the client and the users. For this reason any design approach needs to be reliable, which means that it has to take into account all possible uses as well as hazardous/critical situations. The designers are required to understand the full extent of design information. To better structure the information, formal analysis frameworks are often used. However, the use of formal frameworks could also be inefficient because they tend to be rigid and impose high mental load to the designers.

A design team needs somehow a mechanism to keep everyone on the same level of knowledge, no matter what his or her function is. Every team member needs to know the big picture and the scope of the design assignment. In dealing with the large amount of information, a concrete guidance in making sense of the information is necessary. As a potential solution, different types of information could be combined into situations that give a reasonable coverage of the product use. Designers could then think of these vivid situations as a frame of reference. Nevertheless, they may not know where to start, which combinations to prioritize, whether they have covered all necessary ones, also how to systematically document these combinations of information for easy recall later on.

Creation of scenarios narrows down the information into its essence considerably. Scenarios are a coherent assimilation of information in a form that is easily understood. The tool helps to identify and record combinations of information that make the basis of scenarios (Fig. 5 illustrates this). We also propose to make explicit the relations between the different types of information, as can be seen in Fig. 6 and 7 . This includes the relatedness between the elemental information and scenarios, among scenarios, and between scenarios and requirements. During information use later on, 


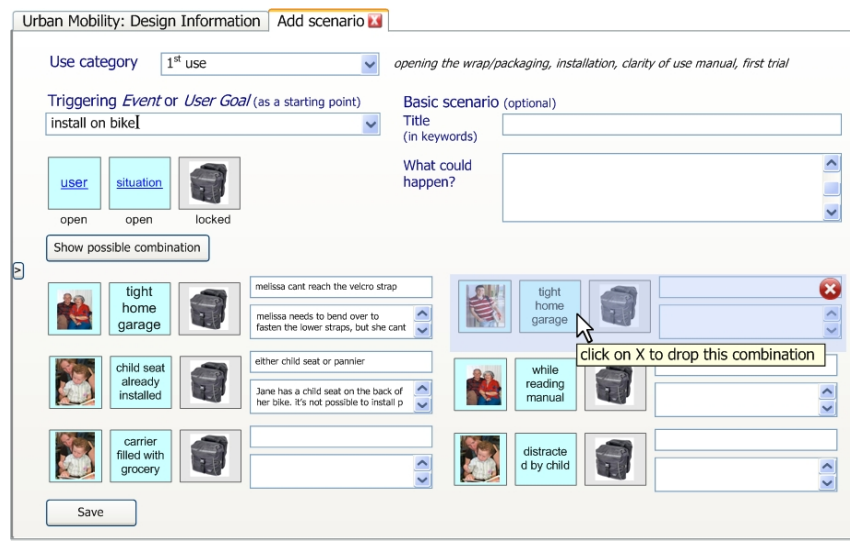

John generates scenarios John starts identifying scenarios that contain important issues. He chooses a common user goal 'installation of product'. Then he pulls out other scenario elements: his main interest being the pannier product type. As John is not sure what other elements would make interesting scenarios, he asks the tool to show him possible combinations of existing information. A few of the combinations trigger John to scribble stories about what could happen.

Fig. 5. Combination of various elements could inspire different scenarios, for which the designer could register short narratives
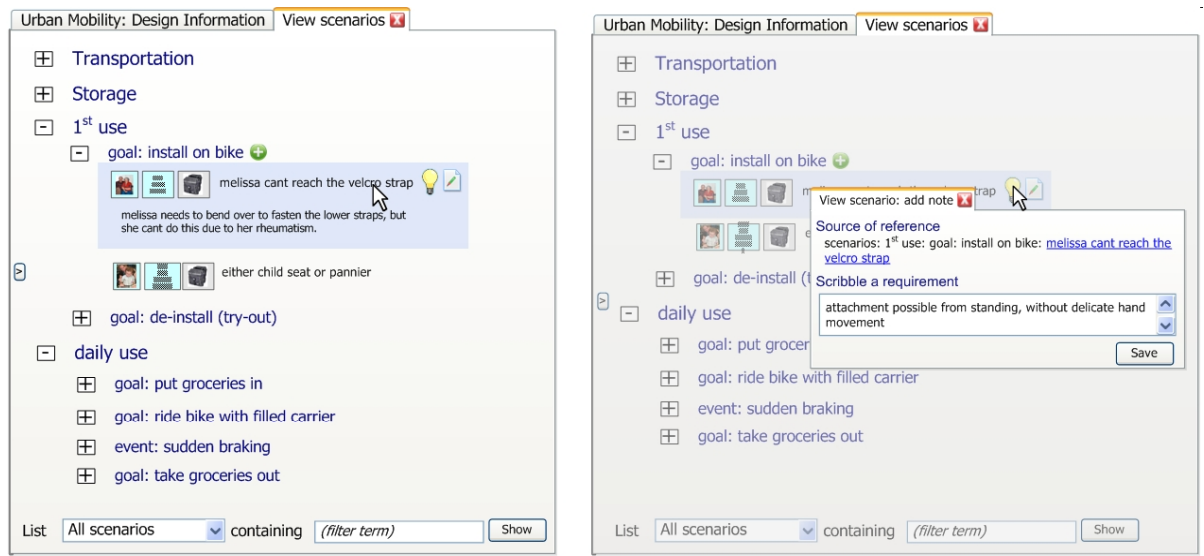

Marie scribbles requirements inspired by the scenarios

Marie scans through the scenarios related to 'installation of the product'. A scenario provokes her mind and she wants to immediately write down her thought. She clicks on the title to highlight the scenario. A set of icons appear next to the scenario title: the light bulb for note-taking and the pen \& paper for editing.

The tool keeps a record on which information has inspired this note, so that Marie can easily find it again for her own reference. After writing down her note, Marie continues scanning the scenarios for inspiration. She knows she can always come back to her scribbled notes later.

Fig. 6. When a scenario inspires the designer, the tool accommodates him or her to readily make notes for tentative requirements

we expect that designers can effortlessly find out relevant information and rationales for scenarios and requirements that have been created. 

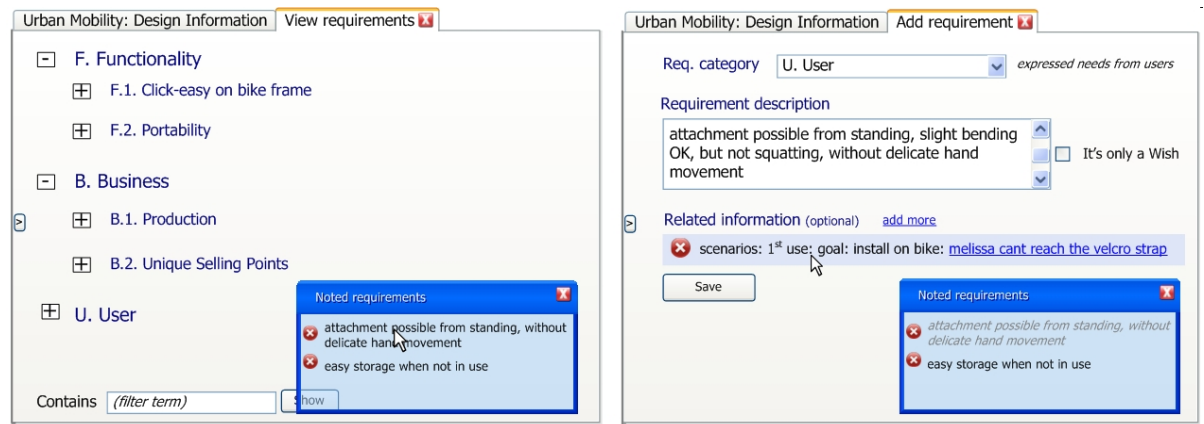

Bob registers a scribbled note as a formal requirement

Bob opens the requirements overview to check out how the team is doing with the analysis. He sees that the 'noted requirements' box now contains new tentative requirements. He clicks one of them about 'attachment (should be) possible from standing' to edit its details. He modifies the description and assigns a proper category for the new requirement, and then saves it. Bob notices that the scenario about Melissa that has inspired this requirement is saved as related info.

Fig. 7. The registration of requirements could take place whenever it is convenient for the designers, therefore not disrupting the creative process by forcing formal tasks in the middle of it

\subsection{Design Information Use Guidance: Integrating Scenarios in the Process}

Scenarios and requirements together are useful as a valid evaluation and reflection tool. While design requirements are often expressed in high-level manner, scenarios identify concrete use situations with explicit elements that advise a proper validation setup. With these potential uses of scenarios and requirements, there needs to be an effortless mechanism to skim, find and relate them to one another.

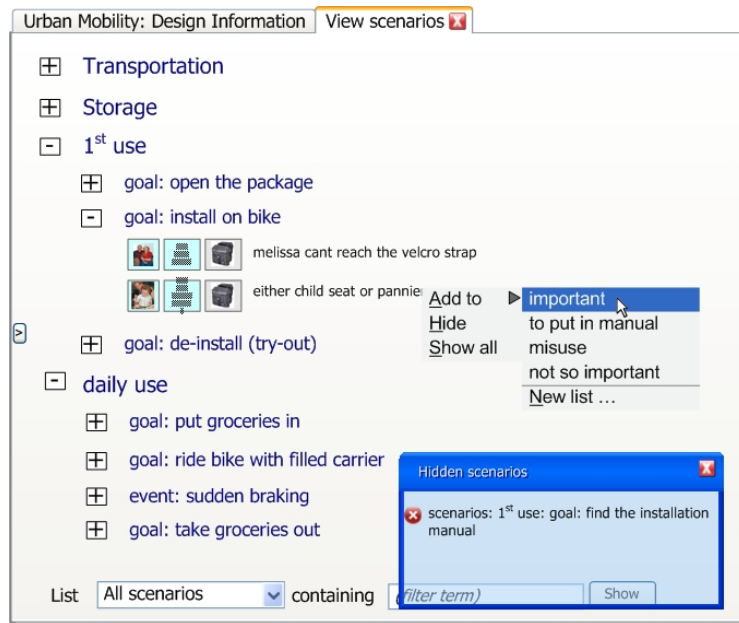

Marie wants to group scenarios on her own categories

Marie studies the scenarios overview. Scenarios about 'finding the installation manual' are not interesting for her; she already hid this scenario group. A small window keeps track of these hidden scenarios.

Marie adds a scenario to the 'important' list; the design team will have to pay special attention to it. Anyone in the design team can easily see the custom lists by choosing from the dropdown in the left-bottom corner.

Fig. 8. Filters and custom sets of scenarios help designers, individually as well as a team, to select and prioritize scenarios according to their main tasks or interests 
Since scenarios are never meant to be the end result of a design process, they need to maintain a connection with other design artifacts. These artifacts could be for instance user requirements, engineering requirements, design concepts, ergonomic or safety standards. Scenarios address mainly the aspects of product use. To relate them with the rest, the design team needs to see the big picture of all the information it has, while at the same time also be able to quickly find a group of related information to examine more closely. This has not been easy in practice, and information sharing among designers and between designers and stakeholders often happens on the spot.

Our tool aims to present an overview of scenarios that is easily grasped by designers. There is a possibility to mark scenarios with specific keywords and priority ranks, to create semi-customized search results for individual design team members (see Figure 8). There should also be different perspectives to view the information as there are multiple functions working together in a design team, as well as varied interests and purposes. The hierarchical structure makes it possible to display compact yet resourceful information.

\section{Discussion and Future Work}

This paper has described our proposal for a tool that serves as an informal framework in a scenario-based product design practice. To conclude our proposal, we reflect upon the suitability of our proposed functionality to solve real design challenges. The first challenge concerns the large amount of design information. The tool puts forward the intention of building and using scenarios throughout the design process, and accommodates this by providing a structure. Designers, as the users, will be guided to single out information that is important as scenario building blocks. Secondly, with all important information neatly documented, the actual benefit lies in the designers being more guided in making sense of the vast amount of information. Designers need to understand a decent multitude of product use situations, without neglecting the critical or extreme situations. By explicitly prompting the designers the combinations of information pieces, the tool inspires them to identify concrete use situations that may lead to crucial design issues. Thirdly, scenarios are not the final results of a design process, rather a helping medium that sums up the designers' knowledge of the design domain. Scenarios therefore cannot be independent from the rest of design artifacts. Integration of scenario uses within the design process however is still largely experimental. Our tool aims to help designers to effortlessly find related information (including scenarios and other information) and to apply filters when needed. With information readily available and well organized, it is easier to employ scenarios and other information for specific purposes, e.g. reflection, validation and decision making. An additional benefit is that designers could use the processed information to generate formal documents e.g. deliveries to third parties.

Our ultimate goal is reached when designers use the proposed tool within their practice because it helps them structure their design knowledge, develop and confirm their rationales, and communicate their ideas and concerns. In achieving this goal, we make sure a close interaction with our stakeholders (i.e. designers and design companies) during the development and later during the evaluation of the tool. 


\section{References}

1. van der Bijl-Brouwer, M., van der Voort, M.C.: Designing for Dynamic Usability: Development of a Design Method that Supports Designing Products for Dynamic Use Situations. Design Principles \& Practices: An International Journal 2(1), 149-158 (2008)

2. Carroll, J.M.: Making Use: Scenario-Based Design of Human-Computer Interactions. MIT Press, London (2000)

3. Rosson, M.B., Carroll, J.M.: Usability Engineering: Scenario-based Development of Human-Computer Interaction. Morgan-Kaufman, San Francisco (2002)

4. Alexander, I.F., Maiden, N. (eds.): Scenarios, Stories, Use Cases: Through the Systems Development Life-Cycle. John Wiley and Sons, New York (2004)

5. Carroll, J.M. (ed.): Scenario-based Design: Envisioning Work and Technology in System Development. John Wiley and Sons, New York (1995)

6. Moggridge, B.: Design by story-telling. Applied Ergonomics 24(1), 15-18 (1993)

7. Suri, J.F., Marsh, M.: Scenario building as an ergonomics method in consumer product design. Applied Ergonomics 31(2), 151-157 (2000)

8. Tideman, M., van der Voort, M.C., van Houten, F.J.A.M.: A Scenario Based Method for Product Design. In: 15th International CIRP Design Seminar, Shanghai, China, pp. 127133 (2005)

9. Cockburn, A.: Using Goal-Based Use Cases. Journal of Object-Oriented Programming 10(7), 56-62 (1997)

10. Sutcliffe, A.G., Maiden, N.A.M., Minocha, S., Manuel, D.: Supporting scenario-based requirements engineering. IEEE Transactions on Software Engineering, Special Issue on Scenario Management 24(12), 1072-1088 (1998)

11. Penna, G.D., Intrigila, B., Laurenzi, A.R., Orefice, S.: An XML environment for scenario based requirements engineering. Journal of Systems and Software 79(3), 379-403 (2006)

12. Rolland, C., Souveyet, C., Achour, C.B.: Guiding goal modelling using scenarios. IEEE Transactions on Software Engineering, Special Issue on Scenario Management 24(12), 1055-1071 (1998)

13. Maiden, N., Minocha, S., Manning, K., Ryan, M.: CREWS-SAVRE: Systematic Scenario Generation and Use. In: 3rd International Conference on Requirements Engineering, Colorado Springs, USA, pp. 148-155 (1998)

14. Shin, J.E., Sutcliffe, A.G., Gregoriades, A.: Scenario advisor tool for requirements engineering. Requirements Engineering 10(2), 132-145 (2005)

15. Lim, Y., Sato, K.: Describing Multiple Aspects of Use Situation: Applications of Design Information Framework to Scenario Development. Design Studies 27(1), 57-76 (2006)

16. Anggreeni, I., van der Voort, M.C.: Tracing the Scenarios in Scenario-Based Product Design: A Study to Understand Scenario Generation. Design Principles and Practices: An International Journal 2(4), 123-136 (2008) 